\title{
Winfriede Schreiber, Chefin des Landesamtes für Verfassungsschutz
}

\author{
Juristinnen machen Karriere - wir stellen sie vor
}

Eine Brandenburger Topjuristin geht nach einem „bunten, aufregenden, kribbelnden“ Berufsleben in den Ruhestand. Das Interview führte die Vorsitzende des djb-Landesverbandes Brandenburg Monika Paulat am 8. März 2013.

\begin{abstract}
Liebe Frau Schreiber, noch sind Sie Chefin des Brandenburgischen Verfassungsschutzes. Zuvor waren Sie Polizeipräsidentin in Frankfurt (Oder). Sie gehen zum 31. Mai 2013 nach einer Verlängerung Ihrer Dienstzeit endgültig in den Ruhestand. Wie ist Ihre Stimmungslage?

Ich freue mich auf den nächsten Lebensabschnitt und empfinde es als neue Herausforderung, den Ruhestand zu gestalten. Traurig muss ich nicht sein, denn ich kann mich über ein erfülltes Berufsleben freuen. Ich erlebe einen schönen, guten Übergang vom Beruf in den Ruhestand, der mir vieles bringen wird, was ich bisher entbehrt oder vernachlässigt habe.
\end{abstract}

Frau Schreiber, Sie kommen aus der Verwaltungsgerichtsbarkeit, waren unter anderem Präsidentin des Verwaltungsgerichts Cottbus, ein vergleichsweise friedliches Terrain. Welche Umstände waren es, die Sie in für eine Frau eher ungewöhnliche Ämter wie die der Präsidentin der Polizei und des Verfassungsschutzes gebracht haben? Haben Sie denn gern Krimis gelesen und Spionagefilme gesehen?

Ja, in der Tat habe ich gern Krimis gelesen, in letzter Zeit aber keine Zeit dafür gehabt. Ich bedaure auch, den neuen James-Bond-Film noch nicht gesehen zu haben. Das sind zum Beispiel Dinge, die ich demnächst intensivieren werde. Aber Spaß einmal beiseite: Die mit der Übernahme des Amtes einer Polizeipräsidentin oder der Leitung des Verfassungsschutzes verbundenen Herausforderungen haben mich sehr gereizt. Im Unterschied zur Tätigkeit einer Richterin, die mit der Anwendung von Gesetzen auf abgeschlossene Lebenssachverhalte sozusagen im Nachhinein reagiert, hatte ich als Polizeipräsidentin und jetzt als Verfassungsschutzleiterin gestalterische Aufgaben. Als ich Polizeipräsidentin in Frankfurt (Oder) wurde, also im Juli 2002, hatte ich es mit der Umsetzung der 1. Polizeistrukturreform in der östlichen Hälfte Brandenburgs zu tun: eine enorme Organisationsaufgabe, die meinem schon während des Jurastudiums vorhandenen verwaltungswissenschaftlichen Interesse entgegenkam. Es ging darum, die Polizei mit möglichst wenig Personal und mit möglichst wenig finanziellen Mitteln zu organisieren, aber auch - und das war mir besonders wichtig - darum, wie man die Reform mit den Menschen, mit den Mitarbeiterinnen und Mitarbeitern gemeinsam verwirklichen kann. Das war und ist ein faszinierender Prozess.

\section{Winfriede Schreiber}

geb. am 22. November 1945 in Berlin

1953-1959 Grundschule Berlin-Spandau

1959-1966 Gymnasium Berlin-Spandau, Abitur

1966-1969 Ausbildung für den gehobenen nichttechnischen Verwaltungsdienst

1969-1972 Verwaltungstätigkeit beim Bezirksamt Charlottenburg, Ernennung zur Stadtinspektorin, später zur Stadtoberinspektorin, Beamtin auf Lebenszeit

1972-1977 Studium der Rechtswissenschaften der FU Berlin. 1. Juristisches Staatsexamen

1977-1980 Referendariat, 2. Juristisches Staatsexamen 1980 Ernennung zur Richterin

1983-1987 Richterin am Verwaltungsgericht Berlin 1987-1989 Abordnung zum Bundesverwaltungsgericht als wissenschaftliche Mitarbeiterin

1989-1992 Richterin am Verwaltungsgericht Berlin

1992-1994 Abordnung zur Senatsverwaltung für Justiz, Justizprüfungsamt

1993 Ernennung zur Richterin am Oberverwaltungsgericht

1994-1998 Versetzung zum Land Brandenburg, Richterin am Oberverwaltungsgericht für das Land Brandenburg

1998-2002 Präsidentin des Verwaltungsgerichts Cottbus 2002-2004 Präsidentin des Polizeipräsidiums Frankfurt (Oder) ab 2005 Leiterin des Verfassungsschutzes Brandenburg

Ich gehe davon aus, dass Ihre Berufswelt in den letzten Jahren männerdominiert war. Wie besteht eine Frau in einer Führungsposition in dieser Männerwelt? Prägt diese männliche Welt die Frau an der Spitze? Oder prägt die Frau die Männerwelt?

Das ist eine gute Frage, die, wenn ich darüber nachdenke, nicht abschließend beantwortet werden kann. Ich habe als Polizeipräsidentin und als Verfassungsschutzchefin in der Tat männerbündische Verhaltensweisen kennengelernt. Von diesen männlich dominierten Verhaltensweisen habe ich mich aber nicht vereinnahmen lassen. Ich habe mich nicht angepasst in der Überzeugung, dass eine weibliche Führungskraft die Kommunikation in einer Behörde positiv zu beeinflussen in der Lage ist. Das Ergebnis ist ein Weniger an Streit und ein Mehr an Ergebnisorientiertheit bei der Aufgabenerfüllung. Frauen haben eine andere Sicht auf die Dinge, sie sind weniger wettbewerbsorientiert und stärker konsensfähig. Das heißt nicht, dass Frauen nicht auch streitig diskutieren könnten oder woll- 
ten. Ihr Ziel ist aber, und das war stets auch meines, eine möglichst hohe Akzeptanz zu erreichen. Jedenfalls denke ich, dass Frauen an der Spitze von Polizei und Nachrichtendiensten diesen Einrichtungen guttun, und so freue ich mich sehr, dass in Niedersachsen gerade eben nach dem Regierungswechsel eine Frau zur Präsidentin des dortigen Verfassungsschutzes ernannt wurde. Die Besetzung der Verfassungsschutzämter mit Frauen ist aber deutlich ausbaufähig, denn insgesamt gibt es mit mir nur drei Verfassungsschutzleiterinnen in der Bundesrepublik. Die Nachrichtendienste sollten und könnten sich durch weibliche Führungskräfte öffnen und die Menschen mitnehmen. Eine Verweiblichung ist auch auf Abteilungs- und
Stärken habe ich an mir selbst schon sehr früh entdeckt. Ich konnte Probleme in die Hand nehmen und nach dem Motto lösen: „Wer kann was? “ Eine meiner Führungseigenschaften ist sicherlich auch die Fähigkeit, Prioritäten zu setzen. Ja, ich meine, Managereigenschaften zu haben. Mein Motto war stets, mit dem, was ich hatte, Ziele zu erreichen. Dazu gehören Mut und Teamgeist. Wenn ich über meine Stärken sprechen soll, dann ist es mein guter Blick für Strukturen und Zusammenhänge und die Fähigkeit wie das Interesse, weit voraus zu denken. Ich habe immer einen Plan B und einen Plan C im Sinne des Polizeiausdrucks „vor der Lage sein“. Und ich schreibe mir die weitere Fähigkeit zu, die Talente

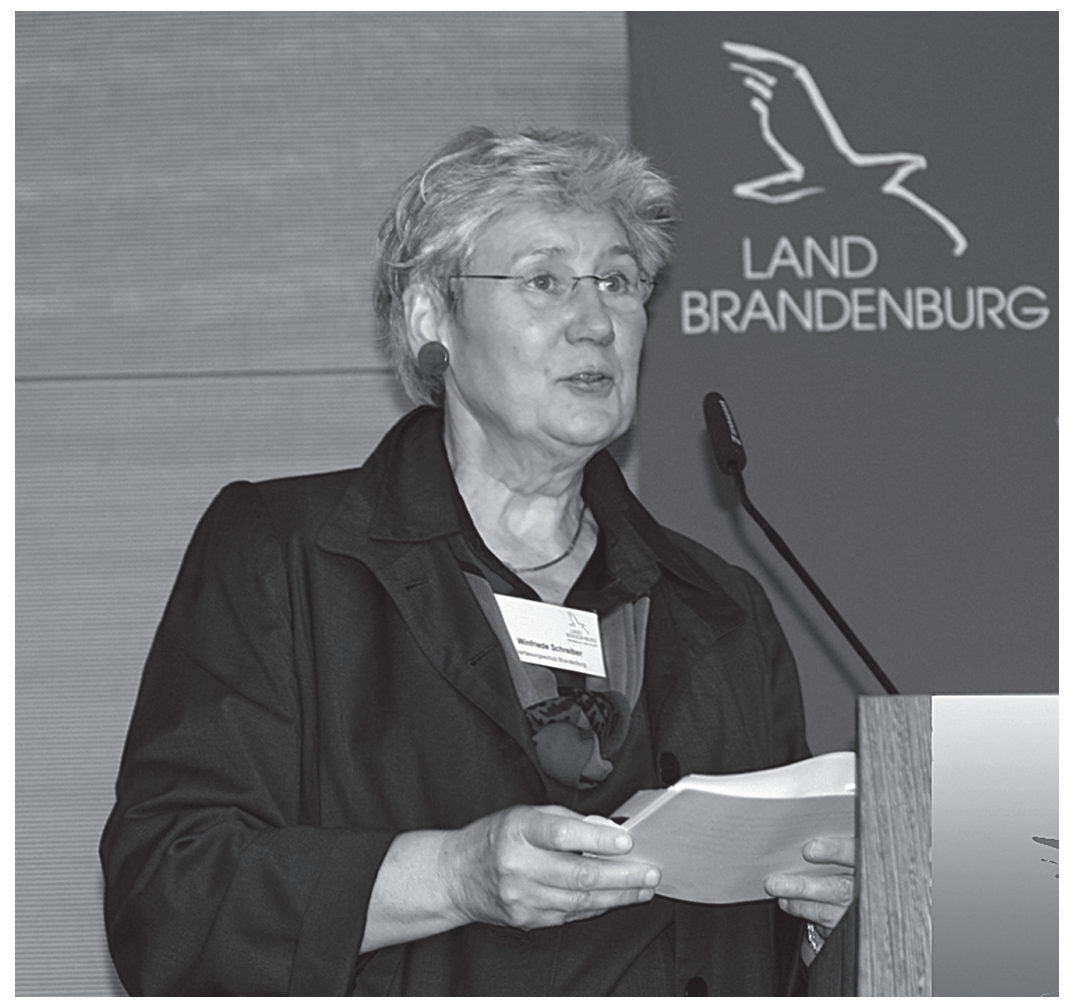

Referatsleiterebene wünschenswert. Immerhin verfügt der Verfassungsschutz des Landes Brandenburg seit Januar 2012 über eine Referatsleiterin. Frauen teilen übrigens gern und bereitwillig ihre Arbeitsergebnisse, was über die Landesgrenzen hinweg durchaus erstrebenswert ist.

\section{Welche Führungseigenschaften rechnen Sie sich zu? Lernt man/frau sie oder hat man/frau sie?}

Ich meine, dass ein gewisses Talent vorhanden sein muss, ein Interesse an der Sache und ein Gestaltungswille. Es muss Bereitschaft bestehen, aus Erfahrung zu lernen und sich zu verbessern. Ich habe viele Coachings und Trainingsmaßnahmen gehabt, die sehr hilfreich sind bei der Bewältigung von Führungsaufgaben. Mit dem dort Gelernten lassen sich Enttäuschungen vermeiden. Wichtig ist in einer Führungsposition die Besinnung auf die eigenen Stärken und die Bereitschaft, andere die Dinge tun zu lassen, die sie besser können als man selbst. Und für besonders wichtig halte ich die Grundüberzeugung, gern mit Menschen zusammenzuarbeiten. Diese von Mitarbeiterinnen und Mitarbeitern gut einschätzen zu können. Sie führt dazu, die richtigen Leute auf den richtigen Platz zu setzen. Mein Motto war immer, die Organisation an die Mitarbeiterinnen und Mitarbeiter anzupassen, und nicht umgekehrt.

\section{Frau Schreiber, sehen Sie einen Unterschied zwischen der Leitung eines Gerichts und der Leitung einer Verwaltungsbe- hörde?}

Bei der Leitung eines Gerichts muss die Kommunikation eine ganz besonders große Rolle spielen. Die Gerichtsleitung hat es mit dem Präsidium als Selbstverwaltungsorgan zu tun. Das ist in einer Behörde anders, obwohl ich hier die Referatsleiter als Kollegium und nicht als untergeordnete Mitarbeiter ansehe. Wichtig ist mir die Feststellung, dass Entscheidungen nicht immer nur nach rein logischen Gesichtspunkten zu fällen sind. Es kommt auch auf subjektive Wahrnehmungen an, die die Entscheidungsfindung beeinflussen können und ihre eigene Logik haben, vielleicht dann eine weibliche. 
Wenn Sie auf Ihr Berufsleben zurückschauen: Was waren die wichtigsten Stationen? Woran denken Sie gern zurück? Was hat Ihnen Kummer bereitet?

Am schönsten waren für mich die Wechsel der Aufgabenbereiche mit immer wieder neuen Herausforderungen. Mein Berufsleben bezeichne ich als bunt, aufregend und kribbelnd. Jede der Stationen war für mich gleich wichtig, und ich glaube nicht, dass irgendetwas vergeblich war. Dieser Abwechslungsreichtum bedeutet für mich eine unendliche Bereicherung meines Lebens. Als Präsidentin des Verwaltungsgerichts Cottbus habe ich die Liebe zur Lausitz entdeckt. Die Tätigkeit als Polizeipräsidentin in Frankfurt (Oder) brachte mir die Begegnung mit Polen. Ich blicke auf ein glückliches und wahrhaft reiches Berufsleben zurück. Von Kümmernissen kann ich nicht berichten. Misserfolge gibt es für mich nicht, nur die Erkenntnis, dass es so, wie ich es dachte, nicht geht und andere Wege zum Ziel führen werden. Ich weiß, dass ich eine anspruchsvolle Vorgesetzte bin, die schon ein wenig sperrig ist. Ich bin sehr gründlich und genau. Aber ich besitze eine hohe Fehlertoleranz. Der griechischen Mythologie entnehme ich, dass nur die Götter fehlerfrei sind. Und das finde ich richtig. Menschen machen Fehler, und das gestehe ich auch meinen Mitarbeiterinnen und Mitarbeitern zu. Deshalb relativiert sich die Charakterisierung als anspruchsvolle Vorgesetzte auf eine ganz menschliche Art und Weise. Ich schätze mich übrigens als Querdenkerin ein, die immer offen ist für neue, kreative Wege.

Wie fällt das berufliche Resümee der Juristin Schreiber aus? War die Vereinbarung von Beruf und Familie ein Thema für Sie? Ich habe eine Tochter, die zu Anfang meines Studiums geboren wurde. Mein Mann war von Anfang an an der Familienarbeit beteiligt, sonst wäre es nicht gegangen. Meine Freude und mein Interesse an Veränderungen hat mein Mann stets geteilt. Für meinen beruflichen Erfolg war das von wesentlicher Bedeutung. Wir haben eine Wochenendbeziehung gelebt, die uns seinerzeit an damals neue Techniken wie das Handy oder den PC herangeführt hat. Beides hat uns während der Woche die zwischen uns bestehenden Entfernungen leichter ertragen lassen.

Frau Schreiber, wir sprechen heute am Weltfrauentag miteinander. Was verbinden Sie mit diesem Tag? Sie sind Mitglied im djb. Warum sind Sie dem djb beigetreten? Und schließlich die Frage: Wofür sollte sich der djb nach Ihrer Ansicht besonders stark machen?

Oh, zunächst habe ich, auf den heutigen Weltfrauentag angesprochen, ein schlechtes Gewissen. Ich wollte heute mit den Frauen meiner Behörde etwas unternehmen. Dies ist aber leider anderen Verpflichtungen zum Opfer gefallen. Die Institution des Frauentages finde ich gut und richtig. Es gibt immer noch genügend Probleme mit der Gleichberechtigung. Die Führungsetagen müssen weiblicher werden. Ich bedaure sehr, dass die bundesdeutsche Gesellschaft auf die Qualitäten von Frauen in großem Stil verzichtet. djb-Mitglied bin ich seit meiner Zeit in Cottbus. Davor stand ich Frauenverbänden eher skeptisch gegenüber. Meine Meinung hat sich aber dann in Cottbus geändert, weil ich feststellte, dass es sehr schwierig war, Frauen in der Gerichtsbarkeit für Beförderungsämter zu gewinnen. Der djb war für mich eine Plattform, dieses Thema zu behandeln. Auch meine Meinung zur Frauenquote habe ich inzwischen geändert. Zur Frage, worum sich der djb über seine jetzigen Themenfelder hinaus noch stärker kümmern sollte, hätte ich einige Vorschläge. Der djb sollte zum Beispiel fragen, wie es den Polizistinnen geht, und die Verstärkung des Verständnisses für den Nachrichtendienst in den Blick nehmen. Da spreche ich natürlich pro domo. Wichtig finde ich aber auch die stärkere Berücksichtigung bildungspolitischer Fragen, insbesondere der berufsbezogenen Bildung. Der djb sollte meiner Meinung nach die frühzeitige Förderung von Führungskräften und die Verzahnung von Bildung und Berufsleben thematisieren. Für ganz wesentlich halte ich dies für den Bereich der Migrantinnen. Und ein Letztes: Ich finde, der djb sollte die Verhältnisse in der Kultur- und Medienpolitik unter die Lupe nehmen. So sind doch Intendantinnen eher rar und Chefredakteurinnen der Presse noch rarer.

Um den Fragenkreis zu schließen: Frau Schreiber, was kommt nach dem letzten Tag des Berufslebens, also nach dem 31. Mai 2013?

Ich will alles nachholen, was ich während der Berufszeit aus Zeitgründen vernachlässigt habe. Ich will Bücher lesen, Filme sehen, in die Oper gehen. Ich möchte Stadtwanderungen unternehmen und zum Beispiel die Veränderungen in Berlin beobachten. Ich bin ein Laufmensch. Und ich möchte reisen. Natürlich werde ich mich auch meiner Familie mehr widmen. Es ist eine endlos lange Liste, die da vor mir liegt und abzuarbeiten ist. Ich bin ein rechter Unruhegeist, muss immer etwas gestalten und Ergebnisse finden. Es muss immer etwas geschafft werden, und wenn es nur das Kochen einer tollen Suppe ist. Sonst fühle ich mich nicht wohl. Und so wird der Ruhestand alles andere als ruhig werden. Wenn meine Liste einmal abgearbeitet sein wird, könnte ich mir auch vorstellen, ehrenamtlich tätig zu sein. Dann würde ich gerne etwas Praktisches und Bodennahes machen wollen. Ich habe da auch schon einige Gedanken, die aber noch nicht spruchreif sind.

Sehr verehrte, liebe Frau Schreiber, ich danke Ihnen sehr herzlich für dieses wunderbar offene, lebhafte Interview, in dem unsere Leserinnen (die Leser natürlich auch) Einblick in Ihr Berufsleben und Ihre Karriere gewinnen konnten. Sie haben uns aber auch mit einer starken Frau bekannt gemacht, die in einer männerdominierten Berufswelt neben Fachkompetenz und Gestaltungswillen stets die Menschen im Blick hat und diese bei der Verwirklichung ihrer Ziele nicht hinter sich lässt, sondern sie - jeder und jede an seinem oder ihrem Platz - mitnimmt. Der djb-Landesvorstand Brandenburg wünscht Ihnen alles Gute für Ihren neuen Lebensabschnitt. Möge er so bunt, aufregend und kribbelnd sein, wie es Ihr Berufsleben war. Wir hoffen für uns, dass Sie auch wieder mehr Zeit haben werden, die Veranstaltungen des djb-Landesverbandes Brandenburg zu besuchen. In diesem Sinne sage ich „Auf Wiedersehen“.

Ja, auch das steht auf meiner Liste. 


\section{European Women Lawyers \\ Association (EWLA)}

\section{Women in Business and Social Development}

13th Annual Congress in Rome

22 November 2013

Hotel Bernini

http://www.ewla.org/News/16257/

http://www.ewla.org/2013ROMECONGRESS/

Programme/

It is with great pleasure that we invite you to the 13th Congress and General Assembly of the European Women Lawyers Association ("EWLA"), which will take place in Rome on 22nd November 2013.

EWLA was founded in 2000 and is a Brussels based international non-profit association. Ever since its founding congress in Berlin, EWLA has aimed to promote fundamental rights and gender equality within law and politics. It has organised events and published statements and resolutions in order to raise the issue of fundamental rights and gender equality into the sight of politicians and law makers. EWLA has become the main network of women from all legal professions all over Europe - and beyond.

Some of the many highlights of EWLA's 13-year history have been the annual congresses that covered a variety of topics relating to gender, law and economics.

Now, after thirteen years of various activities, EWLA is more prepared than ever to raise difficult issues to public awareness. EWLA's Rome Congress will deal with a range of issues from the position of women in the workplace to the impacts of the financial crisis and globalisation, to the importance of gender equality on the European agenda.

EWLA 2013 Congress will measure where Europe stands in terms of progressing on gender balance in law and business and will focus on how redressing gender deficits could positively impact on growth and competitiveness.

The Congress will look both at what the law could change, as well as at what companies and public institutions/ organizations can do to facilitate the economic integration of women, particularly in leadership positions.

Investing in women is the path to progress!

Registration begins on 10th June, 2013 and we welcome all members of EWLA, as well as other European lawyers, to participate so that the congress will be a steppingstone to real gender balance in Europe.

Please pass this invitation on to your colleagues who might be interested in coming to this exciting event.

Please send your registration form (http:// www.ewla.org/2013ROMECONGRESS/ Registration/) to the following address: m.tomsett@gmt-software.be.

For any questions, please contact the Italian board member, Antonia Verna (averna@portolano.it) directly.

We look forward to seeing you in Rome!

Thank you for your support!

Herdís Thorgeirsdóttir

Antonia Verna EWLA President Board Member

\section{Impressum}

\section{Schriftleitung}

Anke Gimbal, Rechtsassessorin (V.i.S.d.P.)

Juliane Lindner

\section{Redaktionsanschrift}

Deutscher Juristinnenbund e. V.

Anklamer Str. 38

10115 Berlin

Telefon: 030 443270-0

Telefax: 030 443270-22

E-Mail: geschaeftsstelle@djb.de

www.djb.de

\section{Erscheinungsweise:}

4 Ausgaben im Jahr

\section{Bezugspreise 2013}

Jahresabonnement 52,- $€$; Einzelheft 14,- $€$ Alle Preise verstehen sich incl. MWSt, zzgl. Vertriebskostenanteil.

Bestellmöglichkeit

Bestellungen beim örtlichen Buchhandel oder direkt bei der Nomos Verlagsgesellschaft Baden-Baden

\section{Kündigungsfrist}

jeweils drei Monate vor Kalenderjahresende

\section{Bankverbindung generel}

Zahlungen jeweils im Voraus an Nomos Verlagsgesellschaft, Postbank Karlsruhe: BLZ 660 100 75, Konto Nr. 73636-751 oder Sparkasse Baden-Baden Gaggenau: BLZ 662500 30, Konto Nr. 5-002266

\section{Druck und Verlag}

Nomos Verlagsgesellschaft mbH \& Co. KG Waldseestr. 3-5, D-76530 Baden-Baden Telefon (07221) 2104-o/Fax (o7221) 2104-27 E-Mailnomos@nomos.de

\section{Anzeigen}

Sales friendly Verlagsdienstleistungen

Pfaffenweg 15, 53227 Bonn

Telefon (0228) 978980, Fax (0228) 9789820

E-Mail roos@sales-friendly.de

Urheber- und Verlagsrechte

Die Zeitschrift sowie alle in ihr enthaltenen einzelnen Beiträge und Abbildungen sind urheberrechtlich geschützt. Jede Verwertung, die nicht ausdrücklich vom Urheberrechtsgesetz zugelassen ist, bedarf der vorherigen Zustimmung des Verlags.

Mit der Annahme zur Veröffentlichung überträgt der Autor dem Verlag das ausschließliche Verlagsrecht für die Zeit bis zum Ablauf des Ur- heberrechts. Eingeschlossen sind insbesondere auch das Recht zur Herstellung elektronischer Versionen und zur Einspeicherung in Datenbanken sowie das Recht zu deren Vervielfältigung und Verbreitung online oder offline ohne zusätzliche Vergütung. Nach Ablauf eines Jahres kann der Autor anderen Verlagen eine einfache Abdruckgenehmigung erteilen; das Recht an der elektronischen Version verbleibt beim Verlag.

Namentlich gekennzeichnete Beiträge geben nicht in jedem Fall die Meinung der Herausgeber/Redaktion oder des Verlages wieder. Unverlangt eingesendete Manuskripte - für die keine Haftung übernommen wird - gelten als Veröffentlichungsvorschlag zu den Bedingungen des Verlages.

Die Redaktion behält sich eine längere Prüfungsfrist vor. Eine Haftung bei Beschädigung oder Verlust wird nicht übernommen. Bei unverlangt zugesandten Rezensionsstücken keine Garantie für Besprechung oder Rückgabe. Es werden nur unveröffentlichte Originalarbeiten angenommen. Die Verfasser erklären sich mit einer nicht sinnentstellenden redaktionellen Bearbeitung einverstanden.

ISSN 1866-377X 